\title{
Discordance between Traditional Pathologic and Energy Metabolic Changes in Very Early Alzheimer's Disease
}

\section{Pathophysiological Implications}

\author{
SATOSHI MINOSHIMA, ${ }^{a, e}$ DONNA J. CROSS, ${ }^{a}$ NORMAN L. FOSTER, ${ }^{b}$ \\ THOMAS R. HENRY, ${ }^{c}$ AND DAVID E. KUHL ${ }^{a, d}$ \\ Departments of ${ }^{a}$ Internal Medicine, ${ }^{b}$ Neurology, and ${ }^{d}$ Radiology, The University of \\ Michigan, Ann Arbor, Michigan 48109, USA \\ ${ }^{c}$ Department of Neurology, Emory University, Atlanta, Georgia 30322, USA
}

\section{INTRODUCTION}

Recent pathologic and metabolic studies of very early Alzheimer's disease suggest a discordance in the distribution between traditional pathologic changes and glucose metabolic reduction. Traditional pathologic changes in very early stages of $\mathrm{AD}$ are shown to occur in the medial temporal lobe, specifically in the entorhinal cortex. ${ }^{1}$ In contrast, very early glucose metabolic reductions occur in the lateral association cortices and posterior cingulate cortex. ${ }^{2}$ This study addresses the possible pathophysiological mechanisms explaining such discordance between metabolic and pathologic changes using positron emission tomography (PET).

\section{GLUCOSE METABOLIC REDUCTION IN VERY EARLY ALZHEIMER'S DISEASE}

\begin{abstract}
A longitudinal study of 23 patients with "isolated memory impairment" identified 10 patients who developed probable AD during a clinical follow-up. Glucose metabolic PET imaging with 2-[ $\left[{ }^{18} \mathrm{~F}\right]$ fluoro-2-deoxy-D-glucose (FDG) was performed for those patients before the clinical diagnosis of dementia was made. Those images were analyzed retrospectively. The mean glucose metabolic map in 10 elderly normal controls showed intense metabolic activity in the posterior cingulate cortex. This activity disappeared in very early AD patients. Statistical subtraction images showed the most severe glucose metabolic reduction in the posterior cingulate cortex followed by the parietotemporal and frontal association cortices, but relatively sparing the primary sensorimotor cortex, occipital cortex, and cerebellum. Glucose metabolism in the medial temporal cortex was also relatively preserved. These findings are contrary to pathological evidence of very early involvement of the medial temporal cortex in AD.
\end{abstract}

${ }^{e}$ Address for correspondence: Satoshi Minoshima, M.D., Ph.D., Department of Internal Medicine, The University of Michigan, B1G412 University Hospital, Ann Arbor, Michigan 481090028. Phone: 734-764-3485; fax: 734-936-8182

e-mail: satoshim@umich.edu 


\section{DOES THE LOSS OF ENTORHINAL EFFERENTS EXPLAIN SEVERE METABOLIC REDUCTION IN THE POSTERIOR CINGULATE AND ASSOCIATION CORTICES IN VERY EARLY AD?}

Neurofibrillary tangles, a traditional pathologic change, occur in a cell body of the neuron. FDG uptake primarily occurs in the region of the synapse. ${ }^{3}$ We hypothesized that the loss of entorhinal efferents caused by AD pathology may have caused glucose metabolic reduction in remote areas such as the posterior cingulate cortex. To test this hypothesis, we compared resting cerebral perfusion scans obtained with $\left[{ }^{15} \mathrm{O}\right]$ water (under the assumption of flow-metabolic coupling) before and after left temporal lobectomy that removed the hippocampus and a substantial portion of entorhinal cortex in nine patients with refractory temporal lobe epilepsy (postoperative $68 \pm 17$ days). Temporal lobectomy resulted in reduced activities in remote areas including the posterior cingulate cortex and thalamus. When compared to the pattern of very early $\mathrm{AD}$, reductions in the posterior cingulate cortex, a part of the thalamus, and a part of the lateral temporal cortex overlapped between two conditions. However, the removal of medial temporal cortex did not result in reduced activities in the parietotemporal and frontal association cortices, where a substantial metabolic reduction was seen in very early AD. In addition, the profile of the magnitude in percent reduction induced by the removal of the medial temporal cortex was different from that observed in very early AD. These findings indicate that severe metabolic reduction observed in the posterior cingulate cortex in very early $\mathrm{AD}$ may be explained in part by the loss of entorhinal efferents due to AD pathology, but more diffuse involvement of association cortices in very early AD cannot be attributed to the entorhinal pathology alone.

\section{DO CHOLINERGIC DEFICITS EXPLAIN SEVERE METABOLIC REDUCTION IN THE CEREBRAL CORTEX IN AD?}

Basal forebrain cholinergic neurons are known to degenerate severely in AD. ${ }^{4}$ We tested a hypothesis that cholinergic deficit may have caused severe metabolic reduction in the cerebral cortex in AD. Acetylcholinesterase activity (AChE) in the brain was measured by PET imaging using $\mathrm{N}$ - $\left[{ }^{11} \mathrm{C}\right]$ methyl-4-piperidinyl propionate $(\mathrm{PMP})^{5}$ and compared to glucose metabolic activity (CMRglc) measured by FDG PET imaging as well as cerebral atrophy measured by 3D Spoiled Gradient Echo Imaging (3D-SPGR) MR imaging in nine AD patients. There was an inconsistency between the patterns of $\mathrm{AChE}$ and CMRglc reduction. Reduction in AChE involved the entire cerebral cortex including the primary sensorimotor cortex. CMRglc reduction and cerebral atrophy primarily involved association cortices sparing the primary sensorimotor cortex. Multivariate correlational analysis (principal components analysis) revealed four latent common factors (eigenvalues > 1) among changes in AChE, CMRglc, and atrophy. The first component was dominated by changes in AChE that had a relatively uniform involvement of the entire cerebral cortex. However, these changes were not intercorrelated with changes in CMRglc or atrophy. The second to fourth components represented intercorrelated changes in CMRglc within the frontal association cortex, parietotemporal cortex, and anterior temopral cortex, respectively. These CMRglc changes were also intercorrelated with atrophy, but not with 
AChE changes. Intercorrelation between changes in CMRglc and atrophy indicates the presence of common pathologic processes that contribute to both measurements.

\section{SUMMARY}

These results suggest that neither the loss of entorhinal efferents nor cholinergic deficit explains all the metabolic features seen in very early AD. Given recent immunohistological evidence of massive glutamatergic synaptic alteration in early AD cortex $^{6}$ and insights into neuronal and glial mechanisms of glucose metabolism, ${ }^{7}$ very early metabolic changes in AD probably reflect a significant impairment of glycolytic activities in the cortico-cortical glutamatergic systems in a preclinical stage of the disease. However, the exact mechanisms of such impairment in these neurons are yet to be determined.

\section{REFERENCES}

1. BraAk, H. \& E. BraAK. 1991. Neuropathological staging of Alzheimer-related changes. Acta Neuropathol. (Berl.) 82: 239-259.

2. Minoshima, S. et al. 1997. Metabolic reduction in the posterior cingulate cortex in very early Alzheimer's disease. Ann. Neurol. 42: 85-94.

3. Kadekaro, M., A.M. Crane \& L. Sokoloff. 1985. Differential effects of electrical stimulation of sciatic nerve on metabolic activity in spinal cord and dorsal root ganglion in the rat. Proc. Natl. Acad. Sci. USA 82: 6010-6013.

4. Whitehouse, P.J. et al. 1981. Alzheimer disease: evidence for selective loss of cholinergic neurons in the nucleus basalis. Ann. Neurol. 10: 122-126.

5. KUHL, D.E. et al. 1999. In vivo imaging of cerebra acetylcholinesterase activity in aging and Alzheimer's disease. Neurology 52: 691-699.

6. Masliah, E. et al. 1991. Cortical and subcortical patterns of synaptophysinlike immunoreactivity in Alzheimer's disease. Am. J. Pathol. 138: 235-246

7. Magistretti, P.J. \& L. Pellerin. 1996. Cellular mechanisms of brain energy metabolism. Relevance to functional brain imaging and to neurodegenerative disorders. Ann. N.Y. Acad. Sci. 777: 380-387 